\title{
Down-regulated G protein-coupled receptor kinase 6 leads to apoptosis in multiple myeloma MM1R cells
}

\author{
ZHIYAO ZHANG ${ }^{1 *}, \mathrm{ZHENYU} \mathrm{LI}^{2 *}$ and WENMING CHEN ${ }^{1}$ \\ ${ }^{1}$ Department of Hematology, Beijing Chao Yang Hospital, Capital Medical University, Beijing 100020; \\ ${ }^{2}$ Department of Hematology, The Affiliated Hospital of Xuzhou Medical College, Xuzhou, Jiangsu 221002, P.R. China
}

Received April 3, 2018; Accepted August 31, 2018

DOI: $10.3892 /$ etm.2018.6722

\begin{abstract}
G protein-coupled receptor kinase 6 (GRK6) is highly expressed in multiple myeloma (MM) cell lines, but absent or only weakly expressed in most primary human somatic cells. In the present study, GRK6 expression was assessed in MM patients and healthy individuals by quantitative polymerase chain reaction. Flow cytometry were performed to measure the apoptosis of lentivial-transfected MM1R cells. Western blot analysis was performed to assess the apoptosis and signal transducer and activator of transcription 3 pathway-related factors. The results demonstrated that GRK6 was differentially expressed in individuals who suffered from MM and healthy individuals. Previous studies have shown that downregulating GRK6 has anti-cancer effects in the MM cell line, MM1R. The present study demonstrated that RNA interference-mediated GRK6 knockdown promoted apoptosis in the MM1R cell line. Therefore, we hypothesized that GRK6 plays a significant role in determining the course of MM.
\end{abstract}

\section{Introduction}

Multiple myeloma (MM) is an age-related monoclonal plasma cell cancer (1). Although recent drug discoveries have greatly improved survival rates, MM currently remains an incurable B-cell malignancy. The initiation and progression of the disease like bone destruction might be caused by the abnormal secretion of related cytokines, activation of oncogenes and molecular genetic abnormalities. Although recent studies

Correspondence to: Professor Wenming Chen, Department of Hematology, Beijing Chao Yang Hospital, Capital Medical University, 8 Gongti South Road, Beijing 100020, P.R. China

E-mail:xybxx@ccmu.edu.cn

*Contributed equally

Abbreviations: MM, multiple myeloma; GRK6, G protein-coupled receptor kinase 6; GPCRs, G protein-coupled receptors

Key words: multiple myeloma, MM1R, GRK6, apoptosis, STAT3 on therapies [such as immunomodulatory drugs (IMiDs) and proteasome inhibitors] have greatly improved clinical outcomes (such as survival, the median survival has increased to over 5 years), MM continues to be an incurable disease (2).

The desensitization mechanism of $\mathrm{G}$ protein-coupled receptors (GPCRs) terminates signaling initiated by $\mathrm{G}$ protein-coupled receptor kinases (GRKs). GRKs are a family of serine/threonine protein kinases; this family of proteins phosphorylates agonist-activated GPCRs specifically. The GRK family is composed of seven members, named GRK1-7, among which GRK2, 3 and 6 are highly expressed in the immune system $(3,4)$. GRKs specifically phosphorylate GPCRs, leading to GPCR uncoupling and the binding of regulatory proteins, such as arrestins; the phosphorylated receptors thus block further activation by $\mathrm{G}$ proteins and initiate receptor internalization $(5,6)$. Thus, GRKs effectively reduce the level of functional receptors on the cell membrane, which results in decreased receptor-mediated signaling. Recent studies have reported that, compared to normal tissues, $G$ protein-coupled receptor kinase 6 (GRK6) is highly expressed in tumor tissues, such as colonic carcinoma (7), liver cancer and medulloblastoma $(8,9)$. Mutations in GRK6 were also detected in gastric cancer (10) and breast cancer. These studies demonstrate that GRK6 might have a certain relationship with tumor occurrence and development, and prognosis.

As previously mentioned, recent studies have shown that GRK6 is involved in many kinds of cell signaling pathways and, to a certain extent, has an impact on many other related cell biology activities, including the regulation of cell apoptosis and the cell cycle. Our previous study showed that down-regulating GRK6 expression can significantly inhibit the proliferation of MM MM1R cells, and stop the cell cycle in the G0/G1 phase by decreasing the expression levels of Cyclin D1 and CDK4. Therefore, we aimed to further investigate the possible role of GRK6 during tumorigenesis in MM cell lines. In this study, the expression of the GRK6 gene was observed in MM1R cells and cell samples isolated from MM patients. Furthermore, this study demonstrated that GRK6 can modulate apoptosis in MM cells.

\section{Materials and methods}

Cell culture. The MM cell lines (NCI-H929, RPMI8226, U266, OPM2, MM1S and MM1R) were obtained from the American 
Type Culture Collection (ATCC, Manassas, VA, USA), and cultured in RPMI 1640 medium supplemented with 10\% fetal bovine serum (both Gibco; Thermo Fisher Scientific, Inc., Waltham, MA, USA). Cell lines were incubated at $37^{\circ} \mathrm{C}$ in a $5 \% \mathrm{CO}_{2}$ atmosphere. GRK6 expression levels were detected in all cell lines (NCI-H929, RPMI8226, U266, OPM2, MM1S and MM1R), and cell functional experiments were performed in MM1R cell subsequently.

MM samples. MM patients and healthy individuals provided informed consent, and the study complied with the Declaration of Helsinki and its amendments. 28 individuals who were admitted to the Affiliated Hospital of Xuzhou Medical College were enrolled in this study, which included 16 patients newly diagnosed with MM in the Department of Hematology from January 2015 to June 2016 and 12 healthy individuals. All subjects met the diagnostic criteria of MM according to the International Myeloma Working Group (IMWG). Bone marrow samples were collected from all $16 \mathrm{MM}$ patients before treatment. Healthy individuals were predominantly bone marrow transplant donors and were not exposed to any known cytotoxic treatment.

Samples from the 16 patients with MM were first treated with Ficoll-Hypaque (GE Healthcare, Shanghai, China) and underwent gradient centrifugation to obtain mononuclear cells. Myeloma cells were isolated from bone marrow by magnetic cell separation (MACS; Miltenyi Biotec GmbH, Bergisch Gladbach, Germany) according to the manufacturer's protocol. The purity of the CD138+MM cells was analyzed by flow cytometry (FACScan cytometer; BD Biosciences, Franklin Lakes, NJ, USA) and determined to be $\geq 90 \%$. The study was approved by the local ethics committee.

Lentiviral vectors and infection. GRK6 specific shRNA sequences were cloned into GV246 lentiviral vectors. Restructured plasmids were named shRNA-1, shRNA-2, shRNA-3 and shRNA-4 (Table I). An empty vector was used as the control plasmid. pSPXA2 and pMD2.G plasmids recombined the restructured lentiviral vectors, and then they were cotransfected into 293FT cells with Lipofectamine 2000 (both Invitrogen; Thermo Fisher Scientific, Inc.). The viral particles were harvested and concentrated by ultracentrifugation. MM1R cells were treated $8 \mu \mathrm{g} / \mathrm{ml}$ polybrene (Sigma-Aldrich; Merck KGaA, Germany) after recombinant virus infection. GFP-positive cells were isolated using puromycin $(0.5 \mu \mathrm{g} / \mathrm{ml})$ after the cells were cultured for 4 days. shRNA- 4 had the highest inhibition rate of GRK6 and was used in the further experiments.

Apoptosis analysis. To assess the level of cell apoptosis, Annexin V PE and 7AAD (both from eBioscience; Thermo Fisher Scientific, Inc.) were used to stain cells according to the manufacturer's instructions. The percentages of early apoptotic cells (Annexin V PE+/7AAD-) and late apoptotic cells (Annexin V PE+/7AAD+) were analysed in this study using CellQuest software in a FACScan cytometer (BD Biosciences).

Western blotting. Protein was isolated using ProteoJET Mammalian Cell Lysis Reagent (Beyotime Institute of
Biotechnology, Haimen, China) according to the manufacturer's instructions. Centrifugation $\left(12,000 \mathrm{x} \mathrm{g}, 15 \mathrm{~min}, 4^{\circ} \mathrm{C}\right)$ was performed to isolate the proteins. They were then denatured and subjected to sodium dodecyl sulfate polyacrylamide gel electrophoresis (SDS-PAGE) and western blotting. Rabbit monoclonal anti-GAPDH (Sigma-Aldrich; Merck KGaA), anti-GRK6 (dilution, 1:1,000, cat. no. 5878), anti-STAT3 (dilution, 1:2,000; cat. no. 4904), anti-pSTAT3 (dilution, 1:1,000; cat. no. 4093), anti-Bax (dilution, 1:1.000; cat. no. 2772) and anti-Bcl-2 (dilution, 1:1,000; cat. no. 2872; all from Cell Signaling Technology, Inc., Danvers, MA, USA) antibodies were used in the present study. We also used horseradish peroxidase-conjugated anti-rabbit secondary antibodies (dilution, 1:1,000; Cell Signaling Technology, Inc.). The resulting bands were visualized using ECL (Thermo Fisher Scientific, Inc.) and densitometric analysis conducted using ImageJ software (v.1.4.3.67; National Institutes of Health, Bethesda, MD, USA).

Reverse transcription-quantitative PCR RT-(qPCR) analysis. Total RNA was extracted from cells and clinical samples using TRIzol reagent (Invitrogen; Thermo Fisher Scientific, Inc.). Complementary DNA was reverse transcribed using $2.5 \mu \mathrm{g}$ of RNA as a template. A Roche LC480 real-time PCR machine and a SYBR Green kit (Applied Biosystems; Thermo Fisher Scientific, Inc.) were used to perform the qPCR analysis. $10 \mu 1$ of the SYBR Green PCR master mix, $50 \mathrm{ng}$ cDNA and $250 \mathrm{nM}$ of each primer were mixed to make up a total volume of $20 \mu \mathrm{l}$, which was used for all PCR experiments. Each sample was analysed in triplicate and the analysis was repeated three times. The thermal cycling conditions were as follows: $10 \mathrm{~min}$ at $95^{\circ} \mathrm{C}$, followed by 40 cycles of $95^{\circ} \mathrm{C}$ for $15 \mathrm{sec}$ and $60^{\circ} \mathrm{C}$ for $60 \mathrm{sec}$. A dissociation curve was used to monitor amplification. The relative expression of the target genes was calculated using the $2^{-\Delta \Delta \mathrm{Cq}}$ method (11). The primer sequences were as follows: GRK6, forward: 5'-CAG CCCATGGAGCTCGAGAAC-3' and reverse: 5'-GGTGCA AAACTGTTAAACGGCGC-3'; GAPDH, forward: 5'-AGA AGGCTGGGGCTCATTTG-3' and reverse: 5'-AGGGGC CATCCACAGTCTTC-3'. GAPDH was used as the endogenous control.

Statistical analysis. The data was analyzed by SPSS statistical software (v.24.0; SPSS, Inc., Chicago, IL, USA), which was expressed as mean \pm standard deviation. Significant differences between groups were determined by Student's t-test. For analyses between more than two groups, Test was performed after the one-way ANOVA and Tukey's test. $\mathrm{P}<0.05$ was considered to indicate a statistically significant difference.

\section{Results}

Expression of GRK6 in MM cell lines. The expression levels of GRK6 mRNA in MM cells lines NCI-H929, RPMI8226, U266, OPM2, MM1S and MM1R were measured using qPCR analysis. The result showed that the GRK6 expression levels varied between the different cell lines and that GRK6 expression was significantly higher in MM1R cells than in the other cells (Fig. 1). 
Table I. GRK6 specific shRNA sequences.

\begin{tabular}{|c|c|c|c|c|c|}
\hline ID & $5^{\prime}$ & Stem & Loop & Stem & $3^{\prime}$ \\
\hline GRK6-1-RNAi-a & Ccgg & ctGAATGTCTTTGGGCTGGAT & CTCGAG & ATCCAGCCCAAAGACATTCAG & TTTTTg \\
\hline GRK6-1-RNAi-b & aattcaaaaa & ctGAATGTCTTTGGGCTGGAT & CTCGAG & ATCCAGCCCAAAGACATTCAG & \\
\hline GRK6-2-RNAi-a & Ccgg & caGTAGGTTTGTAGTGAGCTT & CTCGAG & AAGCTCACTACAAACCTACTG & TTTTTg \\
\hline GRK6-2-RNAi-b & aattcaaaaa & caGTAGGTTTGTAGTGAGCTT & CTCGAG & AAGCTCACTACAAACCTACTG & \\
\hline GRK6-3-RNAi-a & Ccgg & caGCATCTACTTCAACCGTTT & CTCGAG & AAACGGTTGAAGTAGATGCTG & TTTTTg \\
\hline GRK6-3-RNAi-b & aattcaaaaa & caGCATCTACTTCAACCGTTT & CTCGAG & AAACGGTTGAAGTAGATGCTG & \\
\hline GRK6-4-RNAi-a & Ccgg & ccTCGACAGCATCTACTTCAA & CTCGAG & TTGAAGTAGATGCTGTCGAGG & TTTTTg \\
\hline GRK6-4-RNAi-b & aattcaaaaa & ccTCGACAGCATCTACTTCAA & CTCGAG & TTGAAGTAGATGCTGTCGAGG & \\
\hline
\end{tabular}

GRK6, G protein-coupled receptor kinase 6; shRNA, short hairpin RNA; RNAi, RNA interference.

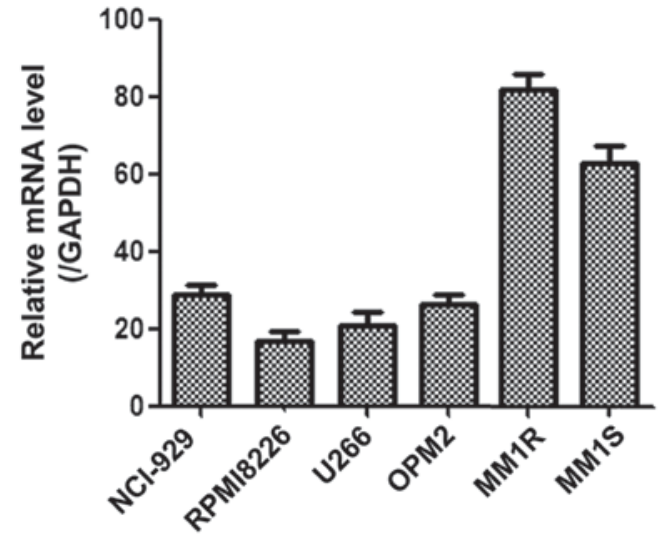

Figure 1. GRK6 expression in MM cell lines. qPCR analysis shows the GRK6 mRNA expression levels in MM cell lines. GRK6, G protein-coupled receptor kinase 6; MM, multiple myeloma.

GRK6 gene expression in patients with MM. We analyzed the expression of GRK6 in $16 \mathrm{MM}$ patients before treatment (Table II). A statistically significant 2-4-fold increase in GRK6 expression was found in the MM patients compared with the healthy individuals $(\mathrm{P}<0.05$; Fig. 2A). Further analyses indicated that GRK6 expression in the MM patients was unrelated to clinical features, such as bone destruction (Fig. 2B), or ISS stage (Fig. 2C) or patient sex (Fig. 2D). The non-significant results may be due to an insufficient sample size.

GRK6 regulates apoptosis in MM1R cells. Decreasing GRK6 expression could significantly lead to the apoptosis of MM1R cells. The MM1R cells stably expressing shRNAs were analyzed by flow cytometry; analyses mainly focused on early apoptotic cells (Annexin V PE+/7AAD-) and late apoptotic cells (Annexin V PE+/7AAD+). A higher rate of late apoptosis was observed in cells treated with the shRNA against GRK6. As demonstrated, GRK6 inhibition is lethal to MM1R cells, especially cells in late apoptosis $(\mathrm{P}<0.01$; Fig. 3$)$.

GRK6 altered the expression of apoptosis-associated proteins. As shown in Fig. 4, the expression of the pro-apoptotic protein, Bax, was increased and an apoptosis-inhibiting protein, Bcl-2, was decreased $(\mathrm{P}<0.05$; Fig. $4 \mathrm{~A}$ and $\mathrm{B})$. Bcl-2/Bax ratios
Table II. Characteristics of patients before treatment.

\begin{tabular}{lc}
\hline Clinical characteristics & $N(\%)(\mathrm{n}=16)$ \\
\hline Age, years & \\
$\leq 65$ & $68(11)$ \\
$\geq 65$ & $32(5)$ \\
Sex & \\
Male & $75(12)$ \\
Female & $25(4)$ \\
ISS stage & \\
I & $31(5)$ \\
II & $25(4)$ \\
III & $44(7)$ \\
Bone destruction & \\
Yes & $75(12)$ \\
No & $25(4)$
\end{tabular}

ISS, international staging system.

indicated that down-regulating GRK6 influenced the apoptosis rate of MM1R cells $(\mathrm{P}<0.05$; Fig. $4 \mathrm{C})$. These results might explain how GRK6 affects the apoptosis signaling pathway in MM1R cells.

GRK6 phosphorylates STAT3 signaling pathway. GRKs serve an important role in the apoptosis signaling pathway in cells by phosphorylating, and thus regulating the activity of, agonist-occupied GPCRs. Here, we attempted to further elucidate these processes by inhibiting the expression of GRK6. GRK6 inhibits apoptosis through a STAT3-dependent mechanism. Phosphorylation of STAT3 plays a crucial role in mediating cell apoptosis and proliferation. Therefore, the effect of GRK6 inhibition on the STAT3 signaling pathway was examined in MM1R cells. As displayed in Fig. 5, the inhibition of GRK6 in MM1R cells using the shRNA resulted in a decrease in STAT3 phosphorylation $(\mathrm{P}<0.05)$. Quantified western blotting results showed that pSTAT3 was significantly decreased, but contradictorily, the concentration of STAT3 increased. 

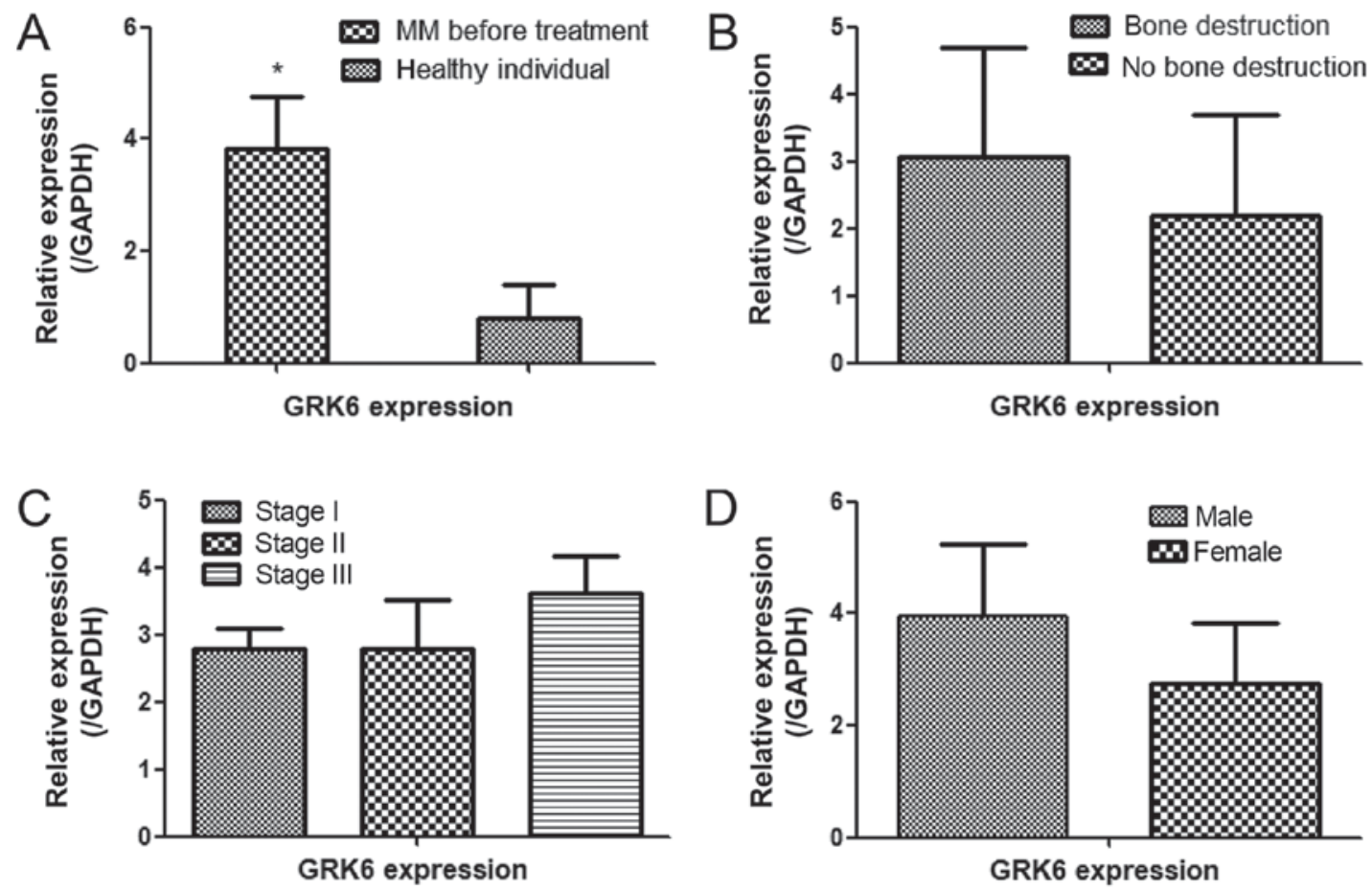

Figure 2. qPCR analysis of the relative expression of GRK6 in MM patient bone marrow. (A) The expression of GRK6 in MM patients before treatment and in healthy controls *. GRK6 expression before treatment was not significantly associated with clinical features when MM patients were stratified according to their (B) level of bone destruction (bone destruction, $n=12$; no bone destruction, $n=4$ ), (C) ISS stage (stage I, $n=5$; stage II, $n=4$; stage III, $n=7$ ) and (D) sex (female, $\mathrm{n}=4$; male, $\mathrm{n}=12$ ) ( $\mathrm{P}<0.05$ vs. the healthy individuals). GRK6, G protein-coupled receptor kinase 6; MM, multiple myeloma.
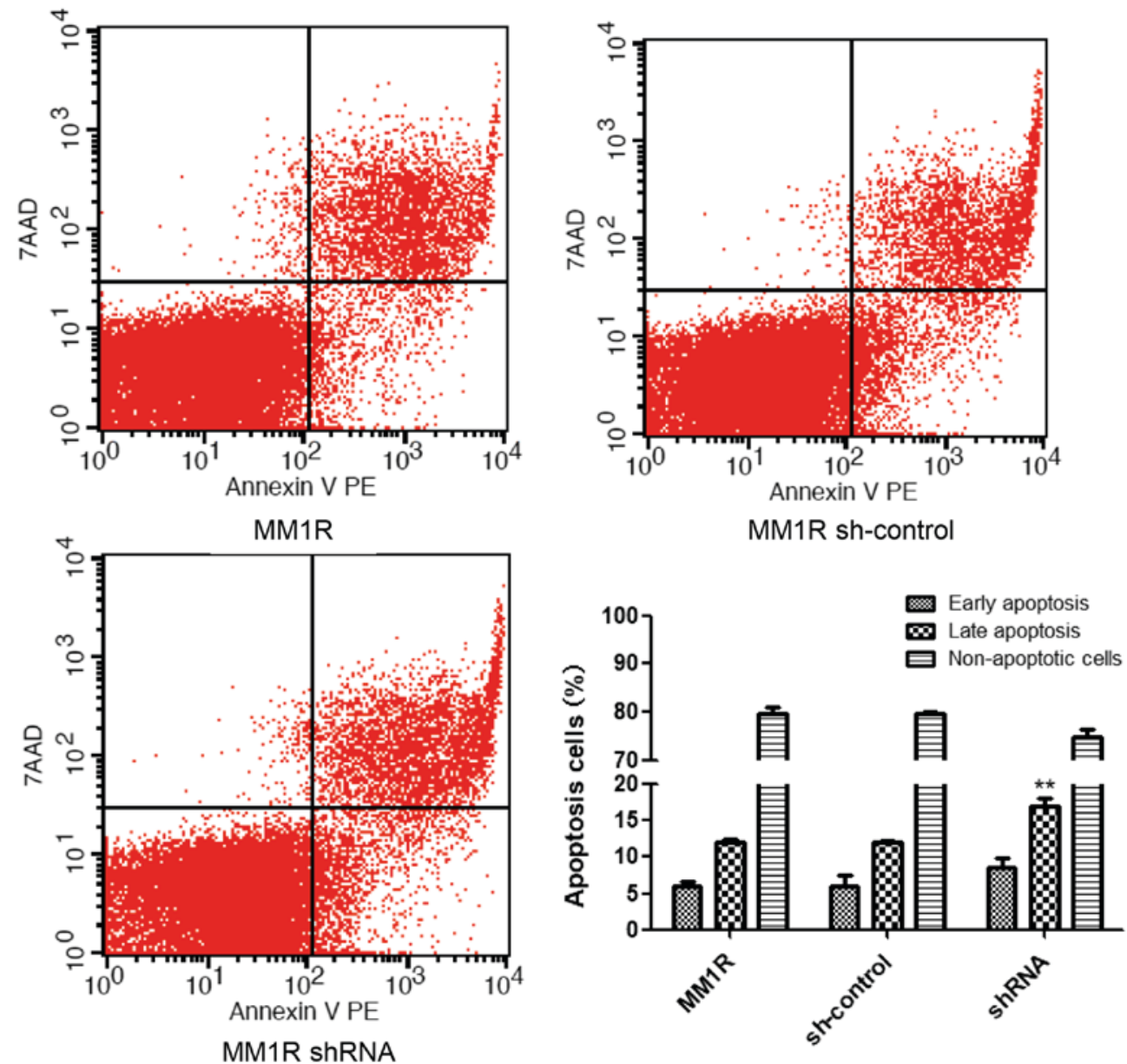

Figure 3. Cell apoptosis was detected in MM1R cells. The apoptotic rate of MM1R cells is shown in bar graphs. Values are presented as mean \pm standard deviation. Data from three independent experiments are shown. ${ }^{* *} \mathrm{P}<0.01$ vs. the sh-control group. 
A
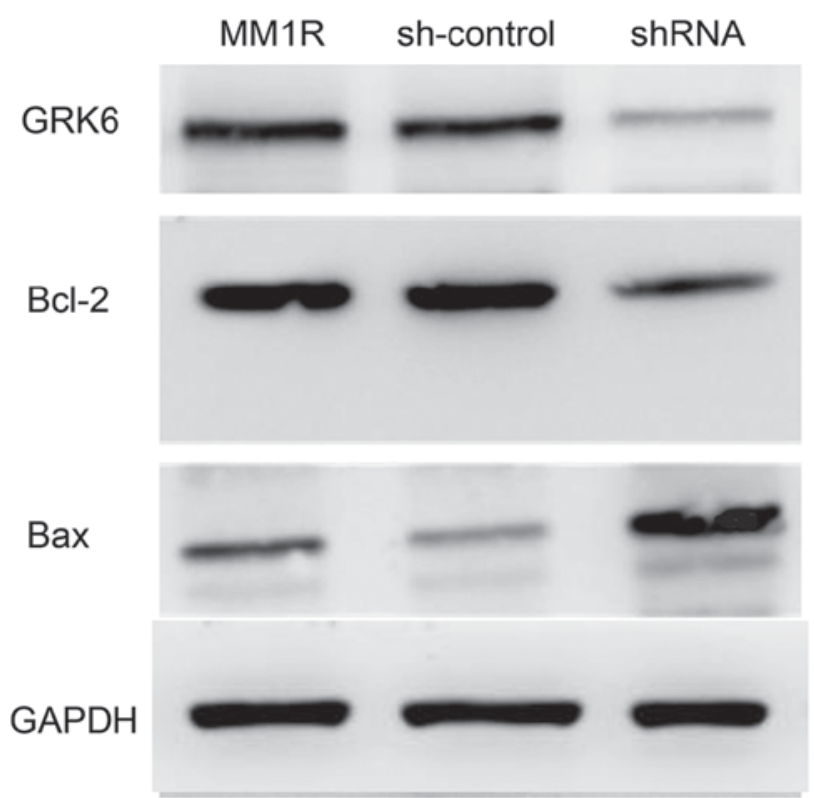

B

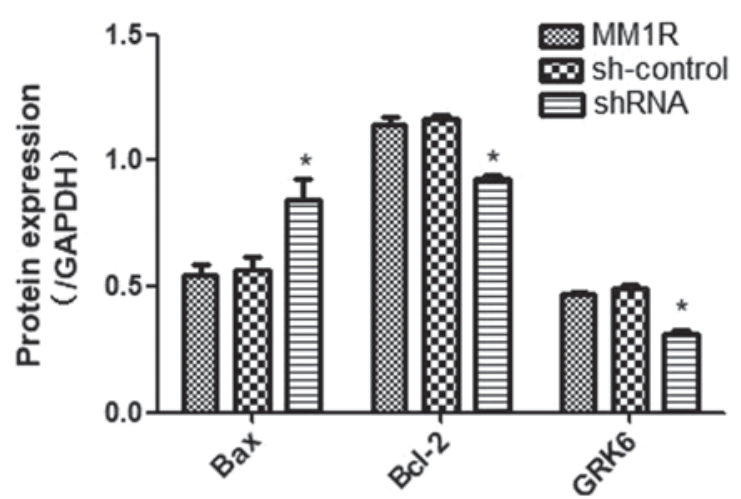

C

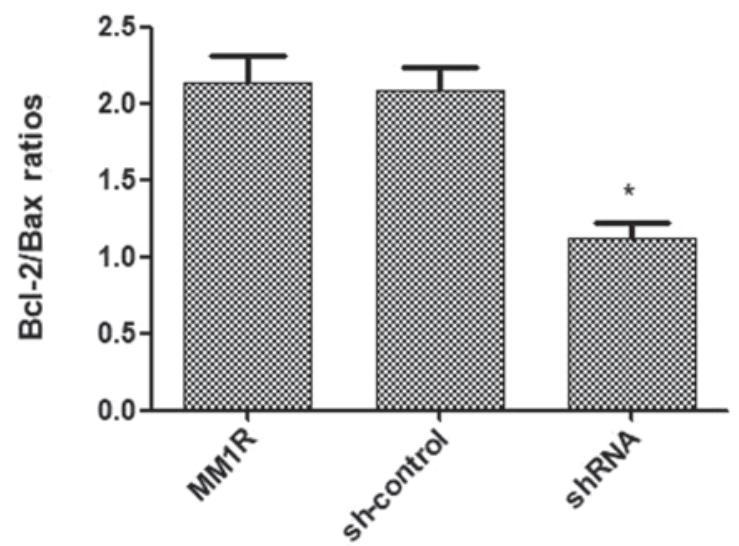

Figure 4. Down-regulated GRK6 affects the expression of the apoptosis-associated proteins. (A) Western blot analysis shows Bcl-2 and Bax protein expression levels. (B) The histogram shows the relative expression levels of Bcl-2 and Bax proteins. (C) The Bcl-2/Bax ratios in different groups. * $\mathrm{P}<0.05$ vs. the sh-control group. GRK6, G protein-coupled receptor kinase 6; Bcl-2, B-cell lymphoma 2; Bax, Bcl-2-associated x protein.
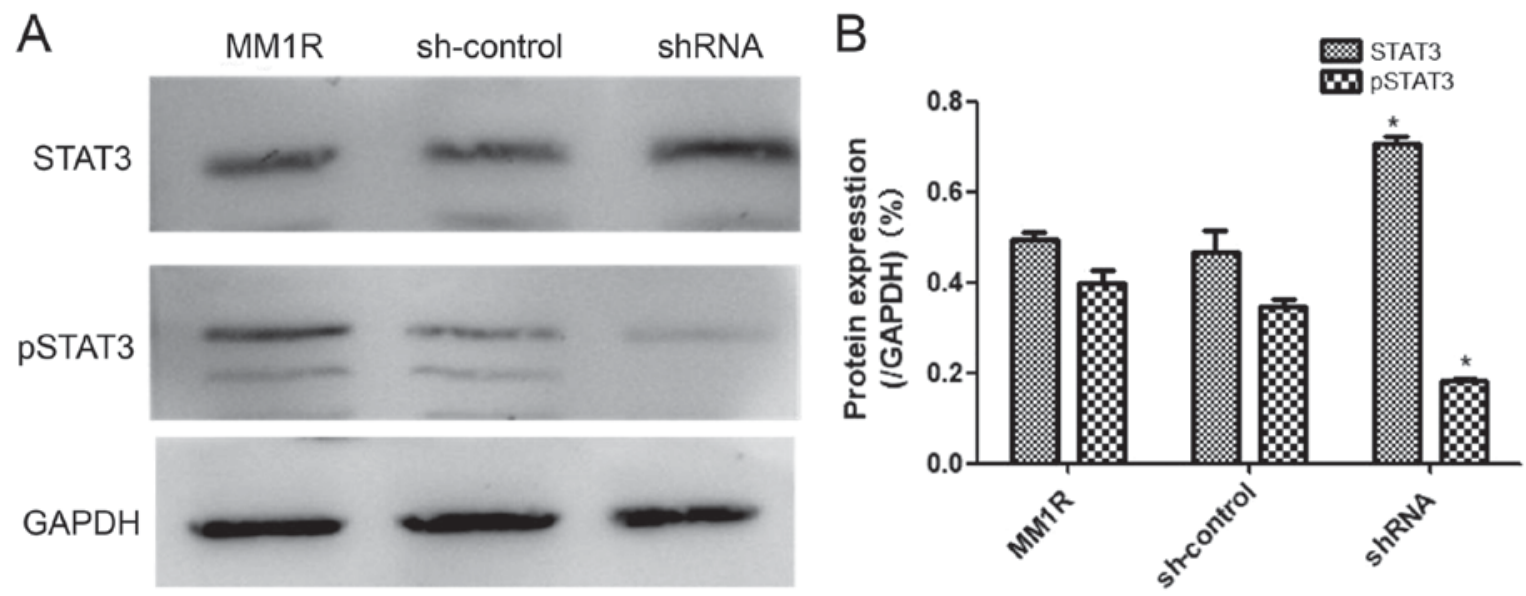

Figure 5. Inhibition of GRK6 alters the levels of the STAT3 protein. (A) Western blot analysis of STAT3 and pSTAT3 protein expression levels. (B) Histogram demonstrating STAT3 and pSTAT3 protein levels. "P<0.05 vs. the sh-control group. GRK6, G protein-coupled receptor kinase 6.

\section{Discussion}

GRK6 is specifically expressed in lymphoid tissues and myeloma, and is absent from or weakly expressed in most primary human somatic tissues; it is similarly expressed in mice (12). The SDF-1/CXCR4 axis (13), and the HSP90 and STAT3 signaling pathway (14-16) play significant roles in MM. Recent studies have shown that GRK6 is involved in many kinds of cell signaling (17). Therefore, multiple signaling pathways might regulate and promote the pathogenesis of MM via GRK6.
Previous studies have shown that GRK6 is highly expressed in colorectal adenocarcinoma, and that gastric carcinoma (18), lung cancer (19) and breast carcinoma (10) might be caused by mutations in GRK6. In our study, compared with healthy individuals, GRK6 was differently expressed in newly diagnosed MM patients, which supports the hypothesis that GRK6 might act as an endogenous promoter of oncogenes. The high expression of GRK6 might inhibit tumor cell apoptosis and promote cell invasion during MM progression.

In a previous study, we found that GRK6 plays an important role in cell proliferation and cell cycle arrest in MM1R 
cells (data not shown). In this study, we verified that GRK6 is extensively expressed in MM cell lines; among which MM1R cells have the highest levels of GRK6 expression. Using the lentivirus containing shRNAs against GRK6, we induced GRK6 knockdown in MM1R cells. As assumed, down-regulating GRK6 induced apoptosis in a large number of MM1R cells. By contrast, the non-silencing lentivirus was nonlethal, demonstrating that inhibiting GRK6 might be lethal to myeloma cells. GRK6 is involved in multiple networks relevant in myeloma, such as EDNRB (20) and CXCR4 (21). STAT3, which is an oncogene $(22,23)$, mediates various cytokines, such as IL6. STAT3 has been shown to play a role in activating myeloma cell lines (24), primary myeloma cells (25) and many other cancer cells (23). Inhibition of the STAT3 signaling pathway in myeloma might lead to both cellular apoptosis and sensitization to chemotherapeutics $(23,26,27)$. In this study, the expression level of phosphorylated STAT3 was significantly decreased. It should be noted that the expression of STAT3 increased after transfection with the shRNA. To elucidate this mechanism, future studies should focus on detecting the level of STAT3 in the cytoplasm and nucleus of cells after transfections with GRK6 shRNA or inhibitors. Notably, a significant decrease in the expression of CDK4 and CyclinD1, which could suppress cell growth, was detected in MM1R cells in which GRK6 was inhibited (data not shown). In addition, we detected the obvious up-regulation of Bax and down-regulation of $\mathrm{Bcl}-2$, which play key roles in the progression of apoptosis in GRK6-deficient myeloma cells. Our results confirmed that the STAT3 signaling pathway is involved in the induction of apoptosis in GRK6-down-regulated MM cells.

Overall, this study postulated that GRK6 might be involved in inhibiting tumor cell apoptosis in MM1R cells. The results indicate that GRK6 may be an important target in the treatment of MM and that GRK6 inhibitors may ameliorate the disease.

\section{Acknowledgements}

We would like to thank all patients for their collaboration in this study. We appreciate the help given by staffs at the Xuzhou Medical College/Laboratory of Transplantation and Immunology for their support during the present study. We also thank all the help given by department of Hematology, Beijing Chao-Yang Hospital.

\section{Funding}

Not applicable.

\section{Availability of data and materials}

All data generated or analyzed during the present study are included in this published article.

\section{Authors' contributions}

$\mathrm{ZZ}$ and WC analyzed and interpreted the patient data, performed the experiments related to the plasma cells, and were major contributors in writing the manuscript. LZ designed the study and was a major contributor in sample collection. All authors read and approved the final manuscript.

\section{Ethics approval and consent to participate}

Patients and healthy individuals provided informed consent, and the study complied with the Declaration of Helsinki and its amendments. The present study was approved by the Clinical Research Ethics Committee of Xuzhou Medical University Affiliated Hospital.

\section{Patient consent for publication}

Not applicable.

\section{Competing interests}

The authors declare that they have no competing interests.

\section{References}

1. Kuehl WM and Bergsagel PL: Multiple myeloma: Evolving genetic events and host interactions. Nat Rev Cancer 2: 175-187, 2002.

2. Rajkumar SV and Kumar S: Multiple myeloma: Diagnosis and treatment. Mayo Clin Proc 91: 101-119, 2016.

3. Chuang TT, Sallese M, Ambrosini G, Parruti G and De Blasi A: High expression of beta-adrenergic receptor kinase in human peripheral blood leukocytes. Isoproterenol and platelet activating factor can induce kinase translocation. J Biol Chem 267: 6886-6892, 1992.

4. Loudon RP, Perussia B and Benovic JL: Differentially regulated expression of the G-protein-coupled receptor kinases, betaARK and GRK6, during myelomonocytic cell development in vitro. Blood 88: 4547-4557, 1996.

5. DeWire SM, Ahn S, Lefkowitz RJ and Shenoy SK: Beta-arrestins and cell signaling. Annu Rev Physiol 69: 483-510, 2007.

6. Gurevich EV and Gurevich VV: Arrestins: Ubiquitous regulators of cellular signaling pathways. Genome Biol 7: 236, 2006.

7. Ek IJ and Otterstad HK: Health care for the aged is better than its start! A status report from communities in Vestfold in 1988-89. Fag Tidsskr Sykepleien 78: 26-28, 1990 (In Norwegian).

8. Kasiske BL: Relationship between vascular disease and age-associated changes in the human kidney. Kidney Int 31: 1153-1159, 1987.

9. Kumar SK, Rajkumar SV, Dispenzieri A, Lacy MQ, Hayman SR, Buadi FK, Zeldenrust SR, Dingli D, Russell SJ, Lust JA, et al: Improved survival in multiple myeloma and the impact of novel therapies. Blood 111: 2516-2520, 2008.

10. Stephens P, Edkins S, Davies H, Greenman C, Cox C, Hunter C, Bignell G, Teague J, Smith R, Stevens C, et al: A screen of the complete protein kinase gene family identifies diverse patterns of somatic mutations in human breast cancer. Nat Genet 37: 590-592, 2005.

11. Livak KJ and Schmittgen TD: Analysis of relative gene expression data using real-time quantitative PCR and the 2(-Delta Delta C(T)) method. Methods 25: 402-408, 2001

12. Fong AM, Premont RT, Richardson RM, Yu YR, Lefkowitz RJ and Patel DD: Defective lymphocyte chemotaxis in beta-arrestin2- and GRK6-deficient mice. Proc Natl Acad Sci USA 99: 7478-7483, 2002.

13. Chudziak D, Spohn G, Karpova D, Dauber K, Wiercinska E, Miettinen JA, Papayannopoulou T and Bönig H: Functional consequences of perturbed CXCL12 signal processing: Analyses of immature hematopoiesis in GRK6-deficient mice. Stem Cells Dev 24: 737-746, 2015.

14. Nieto-Lluis C, Garcia-Pérez M and Cabrera-Santos A: Influence of sympathetic withdrawal on early postinfarction arrhythmias. Acta Physiol Hung 70: 351-356, 1987.

15. Li YP: GRK6 expression in patients with hepatocellular carcinoma. Asian Pac J Trop Med 6: 220-223, 2013.

16. Taipale M, Krykbaeva I, Koeva M, Kayatekin C, Westover KD, Karras GI and Lindquist S: Quantitative analysis of HSP90-client interactions reveals principles of substrate recognition. Cell 150: 987-1001, 2012.

17. Nakaya M, Tajima M, Kosako H, Nakaya T, Hashimoto A, Watari K, Nishihara H, Ohba M, Komiya S, Tani N, et al: GRK6 deficiency in mice causes autoimmune disease due to impaired apoptotic cell clearance. Nat Commun 4: 1532, 2013. 
18. Forbes S, Clements J, Dawson E, Bamford S, Webb T, Dogan A, Flanagan A, Teague J, Wooster R, Futreal PA and Stratton MR: COSMIC 2005. Br J Cancer 94: 318-322, 2006

19. Yao S, Zhong L, Liu J, Feng J, Bian T, Zhang Q, Chen J, Lv X, Chen J and Liu Y: Prognostic value of decreased GRK6 expression in lung adenocarcinoma. J Cancer Res Clin Oncol 142: 2541-2549, 2016

20. Freedman NJ, Ament AS, Oppermann M, Stoffel RH, Exum ST and Lefkowitz RJ: Phosphorylation and desensitization of human endothelin A and B receptors. Evidence for G protein-coupled receptor kinase specificity. J Biol Chem 272: 17734-17743, 1997.

21. Vroon A, Heijnen CJ, Raatgever R, Touw IP, Ploemacher RE Premont RT and Kavelaars A: GRK6 deficiency is associated with enhanced CXCR4-mediated neutrophil chemotaxis in vitro and impaired responsiveness to G-CSF in vivo. J Leukoc Biol 75: 698-704, 2004

22. Bromberg JF, Wrzeszczynska MH, Devgan G, Zhao Y, Pestell RG, Albanese C and Darnell JE Jr: Stat3 as an oncogene. Cell 98: 295-303, 1999.

23. Frank DA: STAT3 as a central mediator of neoplastic cellular transformation. Cancer Lett 251: 199-210, 2007.
24. Brocke-Heidrich K, Kretzschmar AK, Pfeifer G, Henze C, Löffler D, Koczan D, Thiesen HJ, Burger R, Gramatzki M and Horn F: Interleukin-6-dependent gene expression profiles in multiple myeloma INA-6 cells reveal a Bcl-2 family-independent survival pathway closely associated with Stat 3 activation. Blood 103: 242-251, 2004

25. Bharti AC, Shishodia S, Reuben JM, Weber D, Alexanian R, Raj-Vadhan S, Estrov Z, Talpaz M and Aggarwal BB: Nuclear factor-kappaB and STAT3 are constitutively active in CD138+ cells derived from multiple myeloma patients, and suppression of these transcription factors leads to apoptosis. Blood 103: 3175-3184, 2004.

26. Nelson EA, Walker SR, Kepich A, Gashin LB, Hideshima T, Ikeda H, Chauhan D, Anderson KC and Frank DA: Nifuroxazide inhibits survival of multiple myeloma cells by directly inhibiting STAT3. Blood 112: 5095-5102, 2008.

27. Pathak AK, Bhutani M, Nair AS, Ahn KS, Chakraborty A, Kadara H, Guha S, Sethi G and Aggarwal BB: Ursolic acid inhibits STAT3 activation pathway leading to suppression of proliferation and chemosensitization of human multiple myeloma cells. Mol Cancer Res 5: 943-955, 2007. 